\title{
ESTABILIDADE DE CULTIVARES E LINHAGENS DE FEIJOEIRO EM DIFERENTES AMBIENTES NO ESTADO DE SÃO PAULO $\left({ }^{\mathbf{1}}\right)$
}

\author{
SÉRGIO AUGUSTO MORAIS CARBONELL $\left({ }^{* *}, 6\right)$; ALISSON FERNANDO CHIORATO $\left({ }^{2}\right)^{;}$ \\ MARCOS DEON VILELA DE RESENDE ( $\left.{ }^{3}\right)$; LUIZ ANTÔNIO DOS SANTOS DIAS $\left({ }^{4,6}\right)$; \\ ANA LUIZA AHERN BERALDO $\left({ }^{5}\right)$; ELIANA FRANCISCHINELLI PERINA $\left({ }^{5}\right)$
}

\begin{abstract}
RESUMO
O presente trabalho teve como objetivos realizar a avaliação genotípica de linhagens de feijoeiro no Estado de São Paulo pela estimativa dos valores genotípicos das linhagens para cada local e também para o ambiente médio de todos os locais. Estudos da adaptabilidade e estabilidade dos valores genotípicos preditos foram realizados pelo procedimento BLUP. A cultivar Graúna e a linhagem GEN 96A31 apresentaram superioridade média de $10 \%$ sobre a média geral dos 15 ambientes. Os valores foram computados já penalizando as linhagens pela instabilidade através dos locais e ao mesmo tempo capitalizando a capacidade de resposta (adaptabilidade) à melhoria do ambiente, usando propriedades intrínsecas ao método MHPRVG (Média Harmônica da Performance Relativa dos Valores Genéticos). Os métodos MHPRVG, Lin \& Binns e Annicchiarico selecionaram, praticamente, as mesmas linhagens. Contudo, o método MHPRVG forneceu os resultados na própria escala de medição do caráter avaliado, os quais foram interpretados diretamente como valores genéticos para produtividade, estabilidade e adaptabilidade simultaneamente.
\end{abstract}

Palavras-chave: BLUP, MHPRVG, Phaseolus vulgaris, melhoramento, adaptabilidade.

\section{ABSTRACT \\ STABILITY OF COMMON BEAN CULTIVARS AND LINES IN DIFFERENT ENVIRONMENTS IN THE STATE OF SÃO PAULO}

The present study had the objective of a genotypic evaluation of common bean lines in the state of São Paulo. Genotypic values of the lines for each location and for the environment mean of all locations were predicted as well. Studies on the adaptability and stability of the predicted genotypic values were realized by the BLUP procedure. The cultivar Graúna and line GEN 96A31 presented an average superiority of $10 \%$ over the general mean of the 15 environments. These values were computed including the penalization of the lines for the instability across locations and simultaneously crediting the response capacity (adaptability) to improved environments, using intrinsic properties of the MHPRVG method (Harmonic mean of the relative performance of the genetic values). The methods MHPRVG, Lin \& Binns and Annicchiarico selected the same lines. Moreover, the results provided by the method MHPRVG were in the same measurement scale of the evaluated trait and were directly interpreted as genetic values simultaneously, for yield, stability and adaptability.

Key words: BLUP, MHPRVG, Phaseolus vulgaris, breeding, adaptability

( $\left.{ }^{1}\right)$ Recebido para publicação em 4 de novembro de 2005 e aceito para publicação em 21 de novembro de 2006.

$\left({ }^{2}\right)$ Centro de Análises e Pesquisa Tecnológica do Agronegócios dos Grãos e Fibras, IAC, Caixa Postal 28, $13012-970$ Campinas (SP). E-mail: carbonel@iac.sp.gov.br ${ }^{(*)}$ autor correspondente; afchiorato@iac.sp.gov.br

$\left({ }^{3}\right)$ Empresa Brasileira de Pesquisa Agropecuária - EMBRAPA Florestas, 83411-000 Colombo(PR). e-mail: marcosdeon@cnpgc.embrapa.br

$\left({ }^{4}\right)$ Universidade Federal de Viçosa - UFV - BIOAGRO, e-mail: lasdias@ufv.br

$\left({ }^{5}\right)$ Pós-Graduação, Instituto Agronômico de Campinas - IAC, Campinas (SP). e-mail:alberaldo@gmail.com.br; efperina@iac.sp.gov.br

$\left({ }^{6}\right)$ Bolsista em produtividade do CNPq. 


\section{INTRODUÇÃO}

A cultura do feijoeiro no Estado de São Paulo é cultivada em três diferentes épocas de plantio, de acordo com zoneamento ecológico das regiões e épocas de semeadura (feijoeiro das águas, da seca e de inverno), constituindo-se em um sistema quase contínuo de cultivo (PINZAN et al., 1994).

No desenvolvimento de novas linhagens de feijoeiro, convive-se constantemente com o problema da interação genótipo $X$ ambiente $(G \times E)$. Quando se analisa uma série de linhagens em vários ambientes é de se esperar que o desempenho não seja idêntico nos vários ambientes, refletindo as diferentes sensibilidades das linhagens às mudanças ambientais as quais são submetidas (RAMALHo et al., 1993; Carbonell e Pompeu, 2000). A avaliação do desempenho de linhagens em locais estratégicos e dos parâmetros de estabilidade fenotípica permite a identificação de linhagens promissores na região onde são avaliados.

Diante disso, visando à seleção e à recomendação de novas cultivares de feijoeiro, diferentes métodos estatísticos são empregados como Eberhart \& Russel, Lin \& Binns e AMMI, entre outros, para a avaliação de linhagens avançadas (DuARTE e Zimmermann, 1991; Abreu et al., 1992; Carbonell e Pompeu, 1997; e Carbonell et al., 2001).

Para as análises de estabilidade e adaptabilidade, atualmente, procedimentos de interpretação mais simples têm sido preferidos. Assim, medidas que incorporam ambos (estabilidade e adaptabilidade, juntamente com a produtividade) em uma única estatística, tais quais os métodos de AnNicchiarico (1992) e Lin e Binns (1988) e modificações, têm sido enfatizados (Cruz e CARNeIro, 2003). No contexto dos modelos mistos, um método para ordenamento de linhagens simultaneamente por seus valores genéticos (produtividade) e estabilidade, refere-se ao procedimento BLUP sob médias harmônicas (RESENDE, 2002). Quanto menor for o desvio-padrão do comportamento genotípico através dos locais, maior será a média harmônica de seus valores genotípicos através dos locais. Assim, a seleção pelos maiores valores da média harmônica dos valores genotípicos (MHVG) implica simultaneamente seleção para produtividade e estabilidade.

Em termos de adaptabilidade, uma medida simples e eficiente no contexto dos modelos mistos refere-se à performance relativa dos valores genotípicos (PRVG) através dos ambientes. Neste caso, os valores genotípicos preditos (ou os dados originais) são expressos como proporção da média geral de cada local e, posteriormente, obtém-se o valor médio dessa proporção através dos locais. Genericamente, a performance relativa tem sido usada há longo tempo (Wright et al. 1966) em termos de dados fenotípicos e constitui a base do método de AnNICCHIARICO (1992).

A seleção simultânea por produtividade, estabilidade e adaptabilidade, no contexto dos modelos mistos, pode ser realizada pelo método da média harmônica da performance relativa dos valores genéticos (MHPRVG) preditos. Esse método permite selecionar simultaneamente pelos três atributos mencionados e apresenta as seguintes vantagens: (a) considera os efeitos genotípicos como aleatórios e, portanto fornece estabilidade e adaptabilidade genotípica e não fenotípica; (b) permite lidar com desbalanceamento; (c) permite lidar com delineamentos não ortogonais; (d) permite lidar com heterogeneidade de variâncias; (e) permite considerar erros correlacionados dentro de locais; (f) fornece valores genéticos já descontados (penalizados) da instabilidade; (g) pode ser aplicado com qualquer número de ambientes; (h) permite considerar a estabilidade e adaptabilidade na seleção de indivíduos dentro de progênie; (i) não depende da estimação de outros parâmetros tais quais coeficientes de regressão; (j) gera resultados na própria grandeza ou escala do caráter avaliado; (l) permite computar o ganho genético com a seleção pelos três atributos simultaneamente. Estes últimos dois fatores são muito importantes. Outros métodos como o de Lin \& Binns fornecem resultados que não são interpretados diretamente como valores genéticos e, portanto, não permitem computar o ganho genético no caráter composto pela produtividade, estabilidade e adaptabilidade. O método de Annicchiarico depende, adicionalmente, de suposições de valores $\mathrm{Za}$.

Desta forma, o presente trabalho teve como objetivo realizar a avaliação genotípica de cinco cultivares e quinze linhagens de feijoeiro semeadas em 15 ambientes do Estado de São Paulo para as três épocas de cultivo da cultura durante os anos de 2003 e 2004. Foram estimados os valores preditos e genotípicos das linhagens para cada ambiente e também para o ambiente médio de todos os locais, sendo estudado a adaptabilidade e a estabilidade dos valores genotípicos preditos pelo procedimento da melhor predição linear não viesada (BLUP).

\section{MATERIAL E MÉTODOS}

Nos ensaios regionais de VCU do biênio 2003 e 2004 no Estado de São Paulo foram avaliados cinco cultivares e quinze linhagens de feijoeiro representando os avanços tecnológicos dos principais 
programas de melhoramento genético no Brasil (Tabela 1). As cultivares e as linhagens foram avaliadas nas três épocas de semeadura: águas, seca e inverno distribuídos em blocos ao acaso, com quatro repetições.

Os municípios utilizados para semeadura dos ensaios da época da seca foram Capão Bonito $\left(48^{\circ} 36^{\prime} \mathrm{W}-23^{\circ} 50^{\prime} \mathrm{S}\right)$ e Monte Alegre do Sul $\left(46^{\circ} 45^{\prime} \mathrm{W}\right.$

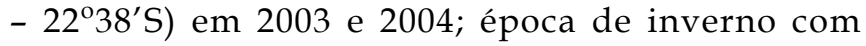
semeaduras nos municípios de Votuporanga $\left(50^{\circ} 11^{\prime} \mathrm{W}\right.$ - $\left.29^{\circ} 09^{\prime} \mathrm{S}\right)$, Ribeirão Preto $\left(47^{\circ} 59^{\prime} \mathrm{W}-21^{\circ} 04^{\prime} \mathrm{S}\right)$ e Pindorama $\left(49^{\circ} 01^{\prime} \mathrm{W}-2^{\circ} 07^{\prime} \mathrm{S}\right)$ em 2003 e 2004, e época das águas, com semeaduras em Capão Bonito, Taquarituba $\left(49^{\circ} 40^{\prime} \mathrm{W}-23^{\circ} 24^{\prime} \mathrm{S}\right)$ e Monte Alegre do Sul em 2003 e por fim, Capão Bonito e Monte Alegre do Sul para o ano de 2004. A parcela experimental foi constituída por quatro linhas de 5,0 metros de comprimento, espaçadas de 0,50 metros entre si, com 10 a 12 plantas viáveis por metro linear. A área útil para avaliação do rendimento correspondeu às duas linhas centrais, não se eliminando 0,50 metros das extremidades de cada linha.

A seleção conjunta por produtividade, estabilidade e adaptabilidade das cultivares e linhagens baseou-se na estatística denominada média harmônica da performance relativa dos valores genéticos (MHPRVG) preditos, conforme descrito por Resende (2004). A MHPRVG conduz a resultados semelhantes aos obtidos pelos métodos descritos por Lin E Binns (1988) e AnNicchiarico (1992) sendo os resultados correlacionados para verificar as proximidades entre as metodologias. Todas as análises foram realizadas por meio do software SelegenReml/Blup (RESENDE, 2002).

\section{RESULTADOS E DISCUSSÃO}

\subsection{Seleção genotípica por local e para o conjunto de ambientes}

Na tabela 2 são apresentados o ordenamento com os respectivos valores genotípicos das linhagens e cultivares avaliadas. Os desvios em relação à média geral foram estimados com base na seleção dos cinco melhores, para o caráter produção, em cada um dos dois locais (Capão Bonito e Monte Alegre do Sul) e também para a análise conjunta dos 15 ambientes. Para um ambiente médio representado pelos 15 ambientes, os cinco melhores foram a cultivar Graúna, as linhagens GEN 96A31, OPS-16, OP-NS-331 e a cultivar TPS-Nobre respectivamente. Ganhos de produtividade da ordem de $4 \%$ podem ser obtidos com a seleção dos dois primeiros genótipos.

Tabela 1. Cultivares e linhagens de feijoeiro avaliadas em 15 ambientes no Estado de São Paulo durante as épocas de cultivo da seca, das águas e de inverno em 2003 e 2004

\begin{tabular}{lcc}
\hline Cultivares e linhagens de feijoeiro & Tipo de Grão & Origem \\
\hline CNFC 9494 & Carioca & EMBRAPA, Goiânia-GO \\
CNFC 9484 & Carioca & EMBRAPA, Goiânia-GO \\
CNFP 10138 & Preto & EMBRAPA, Goiânia-GO \\
CNFP 7966 & Preto & EMBRAPA, Goiânia-GO \\
GEN96A28 & Carioca & IAC, Campinas-SP \\
GEN96A31 & Carioca & IAC, Campinas-SP \\
GEN96A37 & Rosado & IAC, Campinas-SP \\
GEN96A55 & Preto & IAC, Campinas-SP \\
GEN96A102 & Carioca & IAC, Campinas-SP \\
OP-NS-331 & Carioca & UFLA, Lavras-MG \\
OP-S-16 & Carioca & UFLA, Lavras-MG \\
LP98123 & Preto & IAPAR, Londrina-PR \\
LP9820 & Carioca & IAPAR, Londrina-PR \\
LP9963 & Carioca & IAPAR, Londrina-PR \\
Graúna & Preto & IAPAR, Londrina-PR \\
Pérola (Testemunha) & Carioca & EMBRAPA, Goiânia-GO \\
IAC-Carioca Eté (Testemunha) & Carioca & IAC, Campinas-SP \\
IAC- Carioca Tybatã (Testemunha) & Carioca & IAC, Campinas-SP \\
IAC-Una (Testemunha) & Preto & IAC, Campinas-SP \\
TPS-Nobre (Testemunha) & Preto & FT, Ponta Grossa - PR \\
\hline
\end{tabular}




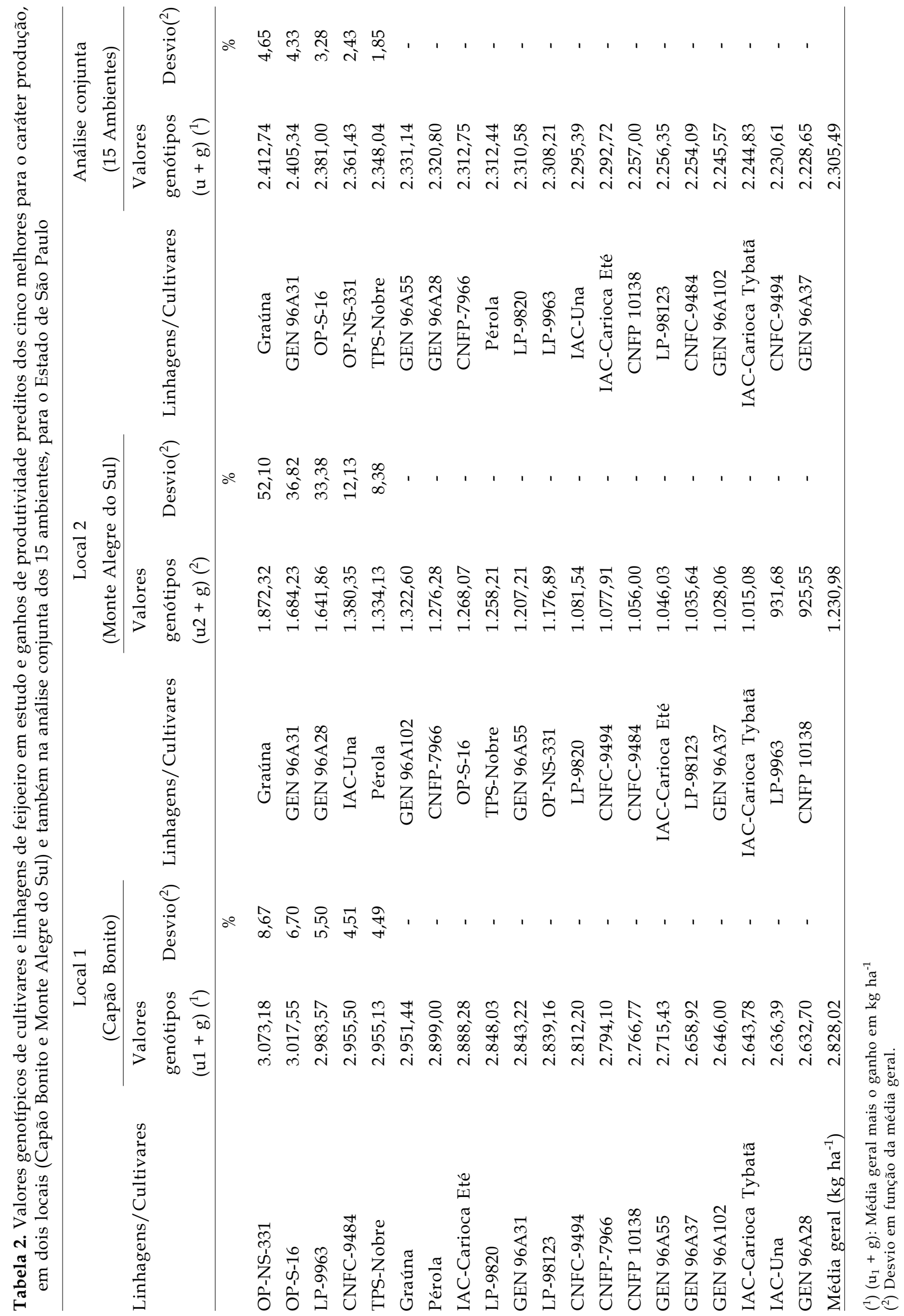


No primeiro local, os cinco melhores foram as linhagens OP-NS-331, OP-S-16, LP-9963, CNFC-9484 e a cultivar TPS-Nobre respectivamente. No segundo local, os cinco melhores foram a cultivar Graúna, as linhagens GEN 96A31, GEN 96A28 e a cultivar Pérola respectivamente. Dentre os cinco melhores, pelo menos dois, em cada local são coincidentes com os melhores na média dos três locais. A seleção por local usa simultaneamente a informação de todos os ambientes de cultivo em relação às diferentes épocas aos anos de cultivo.

\subsection{Seleção conjunta para produtividade, estabili- dade e adaptabilidade}

Na tabela 3, estão apresentados os resultados sobre a estabilidade (MHVG), adaptabilidade (PRVG), e estabilidade e adaptabilidade simultaneamente (MHPRVG), para o caráter produção de grãos.

Verifica-se que os cinco melhores, com base nos critérios PRVG, MHVG e MHPRVG não coincidem com os cinco melhores pelo critério de produtividade média (Tabela 2 ). A coincidência foi de $80 \%$ dentre os cinco melhores e houve inversão de ordem dentre os coincidentes. Esse fato mostra que a utilização desses novos atributos ou critérios de seleção propiciam um refinamento na seleção. Os dois melhores genótipos (a cultivar Graúna e a linhagem GEN 96A31) pelo critério MHPRVG foram superiores em média de $10 \%$ sobre a média geral dos 15 ambientes $\left(2.305,49 \mathrm{~kg} \mathrm{ha}^{-1}\right)$. Esses valores foram computados já penalizando os genótipos pela instabilidade através dos locais e ao mesmo tempo capitalizando a capacidade de resposta (adaptabilidade) à melhoria do ambiente. Essas propriedades são intrínsecas ao método MHPRVG. Os valores de PRVG e MHPRVG (Tabela 3) indicam exatamente a superioridade média do genótipo em relação à média do ambiente em que for cultivado. Assim, o genótipo GEN 96A31 responde em média 1,1063 vezes a média do ambiente em que for plantado. O valor de MHPRVG*MG fornece o valor genotípico médio das linhagens nos locais avaliados, valor já penalizado pela instabilidade e capitalizado pela adaptabilidade.

Dentre as linhagens e as cultivares a serem selecionadas entre as cinco melhores, com base no método da MHPRVG são: a linhagem GEN 96A31, a cultivar Graúna, as linhagens OP-S-16, GEN 96A28 e a cultivar TPS-Nobre. Tal seleção propicia ganho de $8,7 \%$ sobre a média geral dos 15 ambientes, considerando simultaneamente a produtividade, estabilidade e adaptabilidade através dos locais, épocas e anos.

Na tabela 4 são apresentados os resultados referentes à seleção simultânea por produtividade, adaptabilidade e estabilidade por meio do emprego do método de Lin e Binns (1988) sobre os valores genotípicos preditos.

Verifica-se que dentre as 10 melhores cultivares/linhagens selecionadas pela MHPRVG, nove coincidem com as 10 selecionadas pelo método de Lin \& Binns. Nesse método, as melhores linhagens são aquelas com menores valores da estatística Pi, onde $P i \quad\left(V G_{i j} M_{j}\right)^{2} /(2 L)$, sendo $V G_{i j}$ o valor genotípico do genótipo i no local j, Mj é o valor genotípico máximo no local j e L é o número de locais. Tomando por base o método MHPRVG, o único não coincidente foi a cultivar IAC-Carioca Eté, ficando em décimo lugar no ordenamento por esse método e em décimo segundo no método de Lin \& Binns, portanto, em posições muito próximas. As três melhores linhagens foram as mesmas pelos dois métodos.

A correlação estimada entre os parâmetros dos dois métodos foi de alta magnitude $(0,9143)$. O método MHPRVG foi computado em referência à média do ambiente e não em relação ao melhor genótipo em cada ambiente como realizado pelo método de Linn \& Binns. Poderia ter sido computado dessa forma e a concordância entre os dois métodos seria ainda maior.

O método de Annicchiarico foi também computado e houve correlação absoluta nessa mesma magnitude com o método MHPRVG, confirmando que os três métodos utilizam basicamente os mesmos princípios e conceitos. Da mesma forma, CARBONELL et al. (2004) utilizaram um método uni-multivariado para analisar 18 cultivares/linhagens de feijoeiro em 23 ambientes do Estado de São Paulo.

Os parâmetros de estabilidade foram estimados pelos métodos dos Desvios da Produtividade Máxima (MYD) e da análise da Interação Multiplicativa e dos Efeitos Principais Aditivos (AMMI). Os autores concluíram que ambos métodolos produziram resultados semelhantes em termos de identificação das cultivares mais estáveis, porém a maior estabilidade esteve sempre associada à maior produtividade no MYD. O método AMMI além de informar os ambientes facilitou a identificação das cultivares estáveis por inspeção visual. 


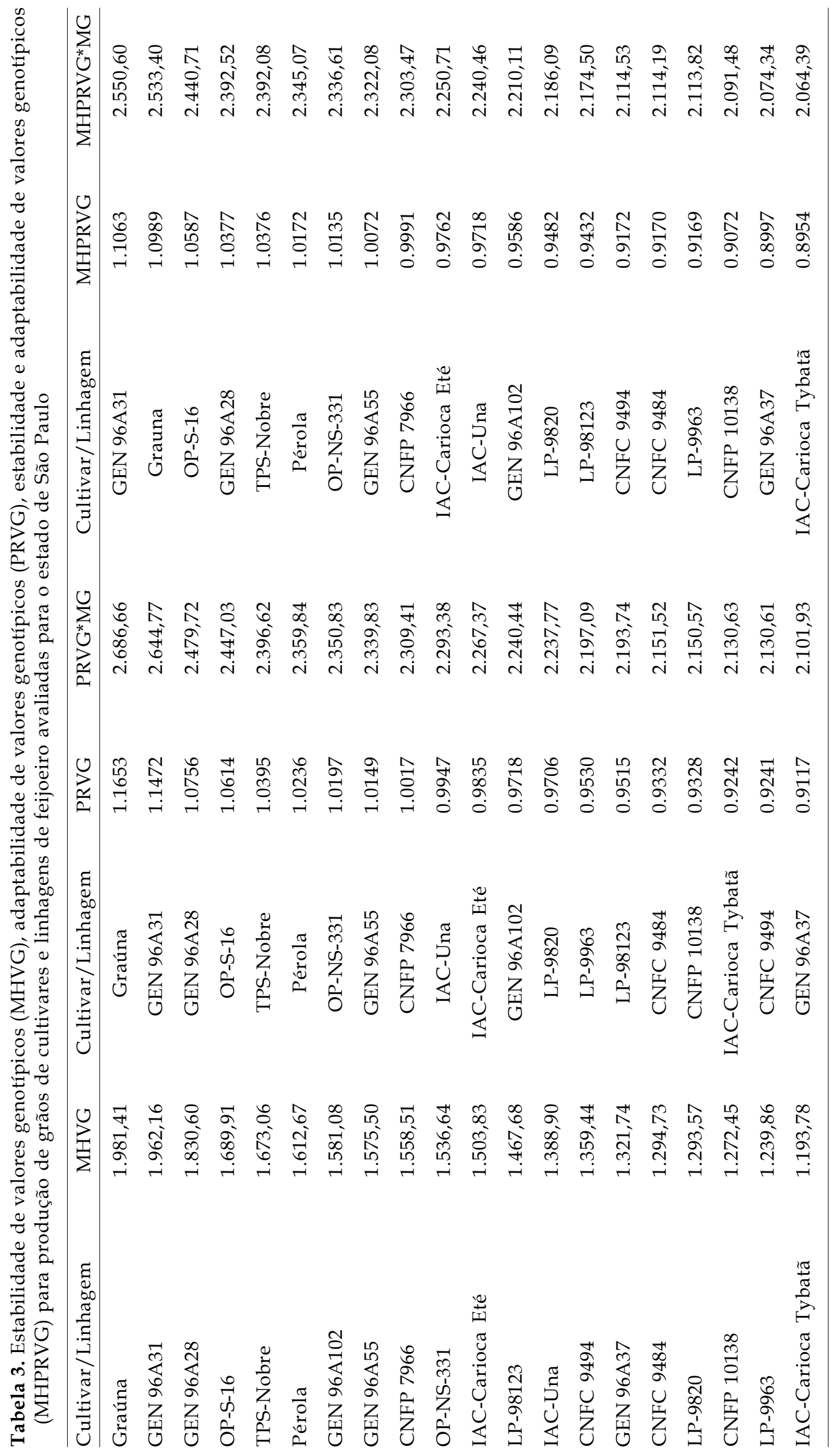


Tabela 4. Estabilidade e adaptabilidade de valores genotípicos para produção em $\mathrm{kg}$ ha ${ }^{(-1)}$ por meio do método (Pi) de LiN E BINNs (1988), referente a cultivares e linhagens de feijoeiro avaliadas durante os anos de 2003 e 2004 no Estado de São Paulo

\begin{tabular}{lr}
\hline Cultivar/Linhagem & \multicolumn{1}{c}{ Pi } \\
\hline Graúna & 12058.32 \\
GEN 96A31 & 15329.12 \\
OP-S-16 & 16914.68 \\
TPS-Nobre & 21494.26 \\
OP-NS-331 & 23792.42 \\
GEN 96A55 & 28425.72 \\
CNFP-7966 & 28913.15 \\
GEN 96A28 & 28983.4 \\
LP-9820 & 30563.59 \\
Pérola & 30964.42 \\
IAC-Una & 36578.01 \\
IAC-Carioca Eté & 37603.78 \\
LP-9963 & 40882.28 \\
GEN 96A102 & 41793.21 \\
LP-98123 & 42302.3 \\
CNFC-9484 & 44697.89 \\
IAC-Carioca Tybatã & 45364.89 \\
CNFP-10138 & 47175.7 \\
CNFC 9494 & 48533.96 \\
GEN 96A37 & 49773.91 \\
\hline
\end{tabular}

O método MHPRVG tem a vantagem de fornecer resultados na própria escala de medição do caráter, os quais podem ser interpretados diretamente como valores genéticos para o caráter avaliado. Esse método permite também calcular o ganho em $\mathrm{kg} \mathrm{ha}^{-1}$ com a seleção simultânea para a produtividade, adaptabilidade e estabilidade, o que não é possível com o método de Lin \& Binns. Assim, a estatística MHPRVG pode ser usada vantajosamente no contexto dos modelos mistos com efeitos genéticos aleatórios.

A consideração dos efeitos genéticos e da interação $\mathrm{G} \times \mathrm{E}$ como aleatórios propicia vantagem também sobre o método AMMI (GAUCH, 1988), o qual trata esses efeitos como fixos e, portanto, atua no nível fenotípico e não genotípico. É importante relatar que o BLUP dos efeitos da interação já elimina os ruídos de tais efeitos, à semelhança do método AMMI, conforme relatado por RESENDE (2004). O método
MHPRVG pode também ser aplicado separadamente para duas classes de ambientes: com índice ambiental negativo e positivo (Tabela 5).

Verifica-se que o ganho em produtividade com a seleção pelos três atributos simultaneamente é maior nos ambientes desfavoráveis (Monte Alegre do Sul seca 2003, Votuporanga - inverno 2003, Monte Alegre do Sul - águas 2003, Capão Bonito - seca 2004, Monte Alegre do Sul - seca 2004, Pindorama - inverno 2004, Ribeirão Preto - inverno 2004, Votuporanga - inverno 2004 e Monte Alegre do Sul - águas 2004) do que nos favoráveis (Capão Bonito - seca 2003, Pindorama inverno 2003, Ribeirão Preto - inverno 2003, Taquarituba - águas 2003, Capão Bonito - águas 2003 e Capão Bonito - águas 2004).

O ganho com a seleção dos três melhores nos ambientes desfavoráveis foi acima de $15 \%$ e nos desfavoráveis, acima de $4 \%$. Dentre os seis melhores, quatro foram coincidentes nos ambientes favoráveis e desfavoráveis sendo a linhagem GEN 96A31, a cultivar Graúna, a linhagem OP-S-16 e a cultivar TPSNobre respectivamente. Duas inversões importantes foram observadas: as linhagens OP-NS-331 e GEN 96A55 foram a primeira e a terceira colocadas nos ambientes favoráveis e apenas a décima segunda e décima nos desfavoráveis respectivamente.

Os métodos que mais penalizaram os valores genotípicos preditos foram pela ordem: MHVG, MHPRVG e PRVG. De maneira genérica, pode-se dizer que os métodos MHVG e MHPRVG são opções seguras, sendo o MHVG um pouco mais conservador.

A linhagem GEN 96A31 e a cultivar Graúna foram às primeiras colocadas em todos os critérios: produtividade, estabilidade e adaptabilidade e nos três atributos simultaneamente em todos os ambientes. Nas demais posições, houve certa alternância de genótipos de acordo com o critério (Tabelas 2 e 3 ).

\section{CONCLUSÕES}

1. A cultivar Graúna e a linhagem GEN 96A31 foram superiores em média de $10 \%$ sobre a média geral dos 15 ambientes.

2. Pelos métodos MHPRVG, Lin \& Binns e Annicchiarico selecionaram-se, praticamente, as mesmas linhagens, no entanto, com o método MHPRVG há a vantagem de fornecer resultados na própria escala de medição do caráter avaliado, os quais podem ser interpretados diretamente como valores genéticos para produtividade, estabilidade e adaptabilidade simultaneamente. 


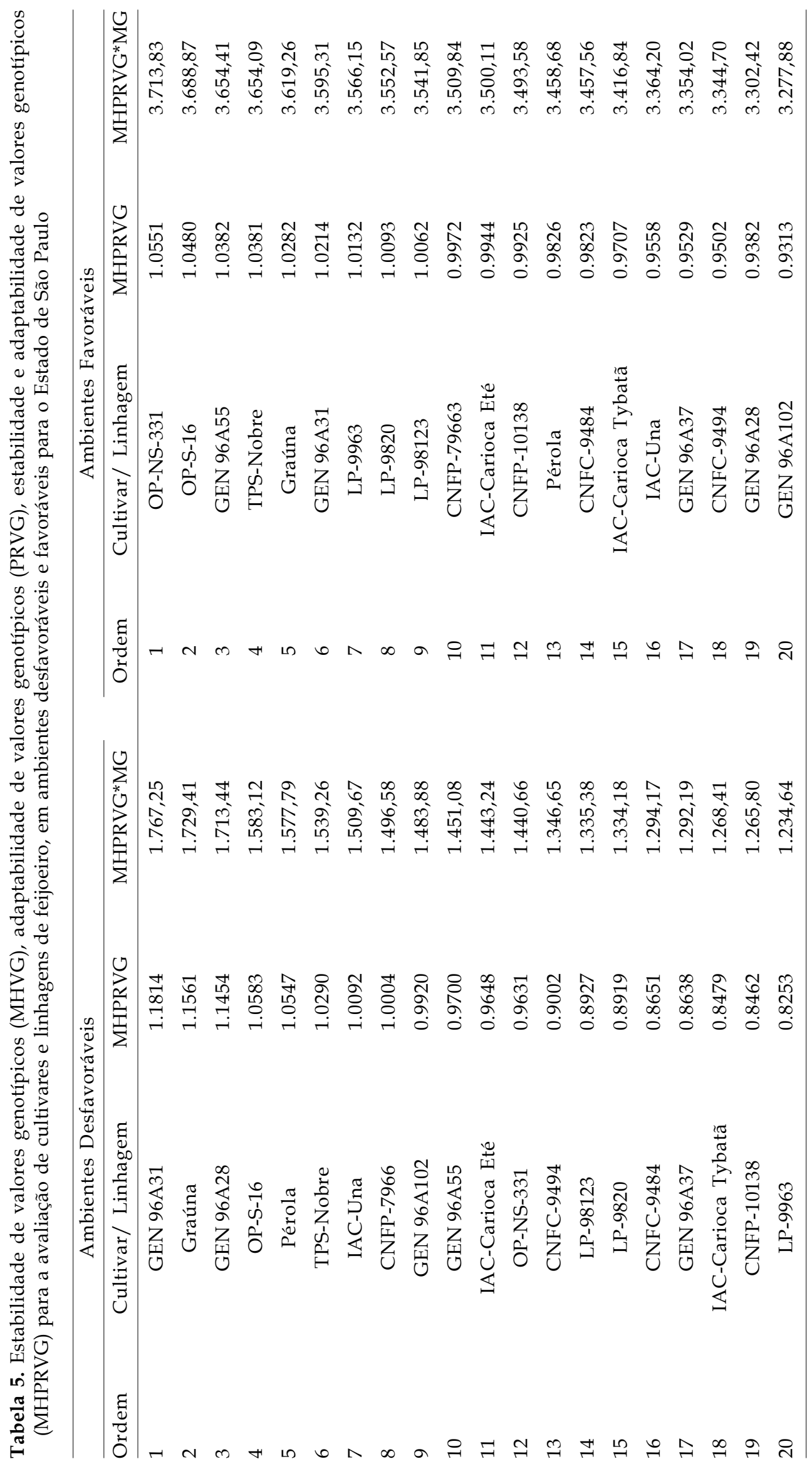




\section{REFERÊNCIAS}

ABREU, A.F.B.; RAMALHO, M.A.P.;SANTOS, J.B. Desempenho e estabilidade fenotípica de cultivares de feijão em algumas localidades do Estado de Minas Gerais no período de 19891991. Ciência e Prática, Lavras, v.16, n.1, p.18-24, 1992.

ANNICCHIARICO, P. Cultivar adaptation and recomendation from alfalfa trials in Northern Italy. Journal of Genetics and Plant Breeding, Rome, v. 46, p. 269-278, 1992.

CARBONELL, S.A.M.; POMPEU, A.S. Estabilidade fenotípica de linhagens de feijoeiro em três épocas de plantio no Estado de São Paulo. Pesquisa Agropecuária Brasileira, Brasília, v.35, n.2, p.321-329, 2000.

CARBONELL, S.A.M.; POMPEU, A.S. Estratificação de ambientes em experimentos de feijoeiro no Estado de São Paulo. Bragantia, Campinas, v.56, n.1, p. 207-218, 1997.

CARBONELL, S.A.M.; AZEVEDO FILHO, J.A.; DIAS, L.A.S.; GONÇALVES, C.; ANTONIO, C.B. Adaptabilidade e estabilidade de produção de cultivares e linhagens de feijoeiro no Estado de São Paulo. Bragantia, Campinas, v.60, n.2, p. 6977, 2001.

CARBONELL, S.A.M.; AZEVEDO FILHO, J.A.; DIAS, L.A.S.; GARCIA, A.A.F.; MORAIS, L.K. Common Bean cultivars and lines interactions with environments. Scientia Agricola, Piracicaba, v.61, n.2, p. 169-177, 2004.

CRUZ, C. D., CARNEIRO, P. C. S. Modelos biométricos aplicados ao melhoramento genético. Volume 2. Viçosa, MG: Editora UFV, 2003. 585p.

DUARTE, J.B.; ZIMMERMANN, M.J. Selection of location for common bean (Phaseolus vulgaris L.) germplasm evaluation. Revista Brasileira de Genética, Ribeirão Preto, v.14, n.3, p.765770, 1991.
GAUCH, H.G. Model selection and validation for yield trials with interaction. Biometrics, Washington, v.44, p.705-715, 1988.

LIN, C.S.; BINNS, M.R. A superiority measure of cultivar performance for cultivar $x$ location data. Canadian Journal of Plant Science, Ottawa, v.68, n. 3, p. 193-198, 1988.

PATTERSON, H. D.; THOMPSON, R. Recovery of inter-block information when block sizes are unequal. Biometrika, London, v. 58, p. 545-554, 1971.

PINZAN, N.R.; BULISANI, E.A.; BERTI, A.J. FEIJÃO: Zoneamento ecológico e épocas de semeadura para o Estado de São Paulo. Campinas: CATI, 1994. 19p. (CATI, Boletim Técnico, 218)

RAMALHO, M.A.P.; ABREU, A.F.B.; RIGUETTO, G.U. Interação de cultivares de feijão por épocas de semeadura em diferentes localidades do Estado de Minas Gerais. Pesquisa Agropecuária Brasileira, Brasília, v.28, n.10, p.1183-1189, 1993.

RESENDE, M.D.V. Genética biométrica e estatística no melhoramento de plantas perenes. Brasília: Embrapa Informação Tecnológica, 2002. 975p.

RESENDE, M.D.V. Métodos estatísticos ótimos na análise de experimentos de campo. Colombo: Embrapa Florestas, 2004. 65p. (Embrapa - Documentos, 100)

WRIGHT, J. W.; PAULEY, S. S.; POLK, R.B; JOKELA, J.J. Performance of Scotch pine varieties in North Central Region. Silvae Genetica, v. 15, p.101-110, 1966. 